Check for updates

Cite this: RSC Adv., 2018, 8, 36722

Received 15th August 2018

Accepted 19th October 2018

DOI: 10.1039/c8ra06836d

rsc.li/rsc-advances

\section{Variably doped nanostructured gallium nitride surfaces can serve as biointerfaces for neurotypic PC12 cells and alter their behavior}

\author{
Patrick J. Snyder, ${ }^{a}$ Pramod Reddy, ${ }^{b}$ Ronny Kirste, ${ }^{b}$ Dennis R. LaJeunesse, (D) ${ }^{c}$ \\ Ramon Collazo ${ }^{a}$ and Albena Ivanisevic (iD) *a
}

\begin{abstract}
Neurotypic PC12 cells behavior was studied on nanostructured GaN and rationalized with respect to surface charge, doping level, and chemical functionalization. The semiconductor analysis included atomic force microscopy, Kelvin probe force microscopy, and X-ray photoelectron spectroscopy. The semiconductor surfaces were then evaluated as biointerfaces, and the in vitro cell behavior was quantified based on cell viability, reactive oxygen species production, as well as time dependent intracellular Ca concentration, $\left[\mathrm{Ca}^{2+}\right]_{\mathrm{i}}$, a known cell-signaling molecule. In this work, we show that persistent photoconductivity (PPC) can be used to alter the surface properties prior to chemical functionalization, the concentration of dopants can have some effect on cellular behavior, and that chemical functionalization changes the surface potential before and after exposure to UV light. Finally, we describe some competing mechanisms of PPC-induced $\left[\mathrm{Ca}^{2+}\right]_{i}$ changes, and how researchers looking to control cell behavior noninvasively can consider PPC as a useful control knob.
\end{abstract}

\section{Introduction}

Today's information-based society has been largely fueled by developments in semiconductor materials. The next generation of emerging technologies will require electronic materials that can confer application-specific properties (chemical, electrical, optical, or a combination thereof). ${ }^{1}$ These properties can be realized through addition of impurity atoms in thin films, ${ }^{2,3}$ changing chemical properties through tethered molecules or films, ${ }^{4-6}$ and topographical pattering of nanostructures or polymers. ${ }^{7-9}$ For optoelectronic devices, understanding the mechanism of doping is the key for both maximizing the efficiency of traditional semiconductor materials, ${ }^{10}$ and enhancing performance and widening applicability and adoption of organic semiconductors, ${ }^{4,11}$ for next-generation applications. Though organic semiconductors have emerged as a strong technology due to the optical gap tunability, the fact that the doping efficiency remains a challenge, sheds light on the complex interactions between the electron transport from organic molecules to inorganic molecules. ${ }^{12}$ This electrical organic-inorganic transport mechanism is crucial for understanding and applying today's semiconductor materials for

${ }^{a}$ Department of Materials Science and Engineering, North Carolina State University, Raleigh, North Carolina, 27695, USA. E-mail: ivanisevic@ncsu.edu

${ }^{b}$ Adroit Materials, 2054 Kildaire Farm Rd., Suite 205, Cary, North Carolina 27518, USA

'Joint School of Nanoscience and Nanoengineering, University of North CarolinaGreensboro, North Carolina A \& T University, Greensboro, North Carolina, 27401, USA wide ranging applications that spans the fields of biology, medicine, and neuroscience. ${ }^{10,13}$

A number of material's properties can be tuned by the addition and concentration of dopants. Conductivity, photoconductivity, optical bandgap, electronic bandgap, dielectric constant, have all been tuned through doping. Small changes in the atomic composition of thin films can lead to great enhancements or deteriorative effects. Doping, in addition to material topography, can lead to changes in a material's optical and electronic properties. For instance, patterning of topographical structures on semiconductor thin films, such as in the case of evanescently coupled nanowire antennas, enhanced the optical read-out efficiency by $10 \times$ as compared to the planar controls. ${ }^{10}$ Doping can also enhance photoconductivity. For example, photoconductive sensitive Al-doped $\mathrm{ZnO}$ has been shown to have enhanced photocurrent and photoconductivity at $1 \% \mathrm{Al}^{14}$ Obviously, the amount of dopants can affect the microstructure. In turn this results in changes of the electron scattering, as in the case of Mg-doped barium strontium titanate. In that case, an increasing amount of $\mathrm{Mg}$ results in the pinning of grain growth, and thus a reduction in overall grain size. Consequently one measures dielectric loss, as well as tunability and leakage current, and dielectric constant reduction from 450 to $205 .{ }^{15}$

Similar to thin films, atomically thin nanomaterials such as black phosphorous and graphene nanoribbons have shown electrical tunability with changes in surface chemistry. For instance, black phosphorous functionalized with aryl diazonium chemistry has exhibited improved p-type doping 
characteristics, which enhances the field-effect transistor mobility and on/off current ratio. ${ }^{16}$ Chemical functionalization with 4-nitrobenzenediazonium and triethylene triamine (DETA) molecules, on graphene nanoribbons has been shown to augment the doping effects and tune the electronic behavior to p- and n-type, respectively. ${ }^{17}$ Furthermore, a large number of studies have focused on targeting biological molecules through various methods such as creating meshed nanowires on the transistor gate to create a real-time non-destructive label-free chemical and biological sensor. ${ }^{18}$ Functionalization of nanoprobes using (Au nanoparticles, quantum dots, magnetic nanoparticles, and carbon nanomaterials) have been shown to especially amenable to biochemical assay applications, resulting effective signal tags or signal amplification systems. ${ }^{19}$ Doping effects however have not been systematically studied with respect to their role in tuning biointerfacial properties.

Previous work conducted by our lab, and others, showed that photoconductive materials such as GaN and porous Si can be used to stimulate biological cells. ${ }^{20-23} \mathrm{GaN}$, unlike $\mathrm{Si}$, once exposed to UV light, shows a corresponding prolonged increase in conductivity lasting for many hours, or even days. ${ }^{24}$ This phenomenon is known as persistent photoconductivity (PPC). In a previous proof-of-concept work, we showed that this PPC along with chemistry and topography, can not only contribute to the localization of cell in a specific locations, ${ }^{23}$ but it can also provide a method for noninvasive in vitro cellular stimulation. ${ }^{25}$ In the referenced work, the properties of persistent photoconductivity (PPC) were utilized to tune the conductivity of a material prior to cellular seeding. It was shown that by exposing $\mathrm{GaN}$ semiconductors, PPC materials, to UV-light, this changes not only the surface potential, but also leads to biological changes in intracellular $\left[\mathrm{Ca}^{2+}\right]$, a key signaling molecule in cellular communication. ${ }^{26}$ In order to quantify this communication, Fluo-4, a commonly-used dye to quantify $\left[\mathrm{Ca}^{2+}\right]_{\mathrm{i}}$ was used. ${ }^{27,28}$ Previous work only quantified the immediate non-invasive cell stimulation. Though the concept was proven, expanding the applicability of these PPC effects requires greater understanding of materials' functionality. Varying the number of charge carriers (dopants) as well as looking at the effect of PPC on chemical functionalization, will provide control knobs for researchers looking to alter cell behavior non-invasively through PPC materials. In this work, we will further examine the following: (1) how can PPC change the surface chemistry prior to chemical functionalization? (2) How does the concentration of dopants affect cellular behavior? (3) How does chemical functionalization affect PPC? and (4) What are the competing mechanisms of PPC-induced $\left[\mathrm{Ca}^{2+}\right]_{\mathrm{i}}$ changes?

In this study, we examined how variably doped semiconductor thin films can lead to distinct in vitro biointerfaces. We relate the dopant dependent properties of the semiconductor thin films before and after exposure to UV light with the observed changes in neurotypic PC12 cell behavior. We utilize 3 different n-type gallium nitride (GaN) thin films with variable Si doping: low-doping (LD) $\left(2 \times 10^{17} \mathrm{~cm}^{-3}\right.$ carriers), medium-doping (MD) $\left(2.1 \times 10^{18} \mathrm{~cm}^{-3}\right.$ carriers), and high doping (HD) $\left(1.5 \times 10^{19} \mathrm{~cm}^{-3}\right.$ carriers). The semiconductor thin film roughness and surface potential were simultaneously analyzed in tapping mode using Atomic Force Microscopy (AFM) and Kelvin Probe Force Microscopy (KPFM). The surface chemistry was examined before and after chemical functionalization with $n$-hexylphosphonic acid with X-ray photoelectron spectroscopy (XPS) with survey and high-resolution scans. In vitro studies with PC12 cells are carried out to understand the role of the variably doped semiconductor thin films on cell viability, reactive oxygen species production as well as time dependent intracellular Ca concentration (referred to as $\left[\mathrm{Ca}^{2+}\right]_{i}$ ).

\section{Experimental section}

\section{Semiconductor thin film growth}

The n-type Ga-polar GaN of three different Si dopant concentrations (Low Doping (LD) $=2 \times 10^{17} \mathrm{~cm}^{-3}$ carriers, Medium Doping $(\mathrm{MD})=2.1 \times 10^{18} \mathrm{~cm}^{-3}$ and High Doping $(\mathrm{HD})=1.5 \times$ $10^{19} \mathrm{~cm}^{-3}$ carriers) were grown on a sapphire substrate by metalorganic chemical vapor deposition using typical precursors: triethylgallium, ammonia, and silane as the precursor for doping. The film thickness of all samples was around $1 \mu \mathrm{m}$. Specific details on the growth of the semiconductor materials are described elsewhere. ${ }^{29,30}$

\section{Chemical functionalization}

The semiconductor samples were sonicated in acetone for $20 \mathrm{~min}$ and then sonicated in methanol for $20 \mathrm{~min}$. The 'Cleaned' samples were removed and dried with $\mathrm{N}_{2}$ gas. The functionalized samples were then placed in a 50/50 vol\% of $100{ }^{\circ} \mathrm{C} \mathrm{H}_{2} \mathrm{O} / \mathrm{HCl}$ for $20 \mathrm{~min}$. The samples were then rinsed with DI water, and placed in a 50/50 vol\% $3 \mathrm{mM} n$-hexylphosphonic acid/60\% phosphoric acid $\left(\mathrm{H}_{3} \mathrm{PO}_{4}\right)$. After functionalization, the samples were dried using $\mathrm{N}_{2}$ gas.

\section{AFM \& KPFM}

The roughness was quantified using an Asylum Cypher S AFM. At least nine $5 \times 5 \mu \mathrm{m}$ scans in AC air mode containing topography, phase, and amplitude information. The RMS was quantified using Asylum Research software running Igor Pro (version 13.01.68). The Pt probes coated with Ir were obtained through Asylum Research were specifically designed for electrical measurements with $f=350 \mathrm{kHz}$. A soft-contact approach was used, and the probe was electrically driven with a signal that was equal to the natural resonance of the cantilever $(\sim 350$ $\mathrm{kHz}$ ) with an amplitude of $3 \mathrm{~V}$. The sample was attached to a sample holder using conductive tape; additionally metallized contacts were placed on the samples and connected to the onboard AFM ground contact.

\section{XPS}

The surface chemistry of clean and functionalized GaN samples were analyzed with a Kratos Axis Ultra DLD X-ray photoelectron spectrometer. Three spots on three samples for each doping level (HD, MD, and LD) and chemistry (clean, functionalized), were used ( 9 total spots) for a fully factorial experimental design where high resolution scans (pass energy $=20 \mathrm{eV}$ ) of $(\mathrm{C} 1 \mathrm{~s}, \mathrm{Ga}$ 2p, Ga 3d, P 2p, N 1s, O 1s) were obtained with a wide survey 
scan (pass energy $=160 \mathrm{eV}$ ). Casa XPS v2.3.12.8 was used for determining atomic percentages of each sample from the survey scans. Background subtraction was done using a Shirley approximation and all peaks were calibrated by setting the $\mathrm{Ga}$ $3 \mathrm{~d}$ peak to $19.6 \mathrm{eV}$ to avoid errors due to carbon contamination. ${ }^{31}$

\section{In vitro cell growth and assays}

Second passage PC12 cells (ATCC) were grown to $~ 90 \%$ confluency in collagen coated Petri dishes. After trypsin-EDTA was added to encourage cellular detachment, and the plates were incubated for $20 \mathrm{~min}$ in a $37{ }^{\circ} \mathrm{C}, 5 \% \mathrm{CO}_{2}$ environment. The cellular detachment was confirmed through optical microscopy, and the cell suspensions were centrifuged at $200 \mathrm{~g}$ for $8 \mathrm{~min}$. Cell media was prepared to the following concentration: DMEM containing $12.5 \%$ horse serum, $2.5 \%$ fetal bovine serum, and $1 \%$ penicillin/streptomycin. This cell media was prepared at the beginning of the experiment and used throughout the seven-day experiment. 24 well plates were previously prepared and coated with collagen I. The working volume during cell incubation was maintained at $1 \mathrm{ml}$ of cell media. After 1 day (18 hours after day 0 (cell seeding)) of growth in cell media, the cell media was replaced with DMEM supplemented with 1 vol\% horse serum and $50 \mathrm{ng} \mathrm{ml} \mathrm{m}^{-1}$ of neuronal growth factor (NGF). This NGFDMEM media was then used for the remainder of the experiment. Three types of assays were done: Alamar Blue $(\mathrm{AB})$ for cell viability, DCFH-DA for quantification of reactive oxygen species (ROS) and Fluo-4 Ca assay to measure intracellular calcium, $\left[\mathrm{Ca}^{2+}\right]_{\mathrm{i}}$. The $\mathrm{AB}$ assay was performed first, and $100 \mu \mathrm{l}$ was added to the $1 \mathrm{ml}$ working volume of cells media, and the plates were incubated for 4 hours ${ }^{\circ} \mathrm{C}, 5 \% \mathrm{CO}_{2}$ environment. Six replicates of $150 \mu \mathrm{l}$ volume of each well were transferred to a 96 well plate after the incubation. A Tecan GENios microplate reader was used to take $\mathrm{AB}$ absorbance measurements at $570 \mathrm{~nm}$ and $600 \mathrm{~nm}$. The data was normalized with positive (cells + no semiconductor) and negative controls (no cells + no semiconductor) for each day. After the remnant media was aspirated from the wells, $0.5 \mathrm{ml}$ of cell media was added to each well along with $0.5 \mathrm{ml}$ of a DCFH-DA - PBS solution $\left(10 \mu \mathrm{g} \mathrm{ml}{ }^{-1}\right)$. The solution was incubated for $1 \mathrm{~h}$, and six replicates were transferred to a 96 well plate for measurement $\left(\lambda_{\mathrm{Ex}}=485 \mathrm{~nm}, \lambda_{\mathrm{Em}}=\right.$ $535 \mathrm{~nm}) .300 \mu \mathrm{l}$ of the Fluo-4 Ca assay dye and included buffer solution (without probenecid) was added to each well along with $100 \mu \mathrm{l}$ of cell media. Six replicates were transferred into a 96 well plate for microplate measurement $\left(\lambda_{\mathrm{Ex}}=492 \mathrm{~nm}, \lambda_{\mathrm{Em}}\right.$ $=535 \mathrm{~nm}$ ). For each set of measurements, the replicates were averaged and analyzed using a 3-way ANOVA. All of the statistical analyses were performed using OriginPro 2016 (b9.3.1.273). Three-way ANOVAs were used to find significant differences between the experimental conditions with $\alpha=0.05$.

\section{Results and discussion}

\section{Surface topography characterization}

The semiconductor roughness was quantified using AFM (ACAir) in order to measure the topographical changes with

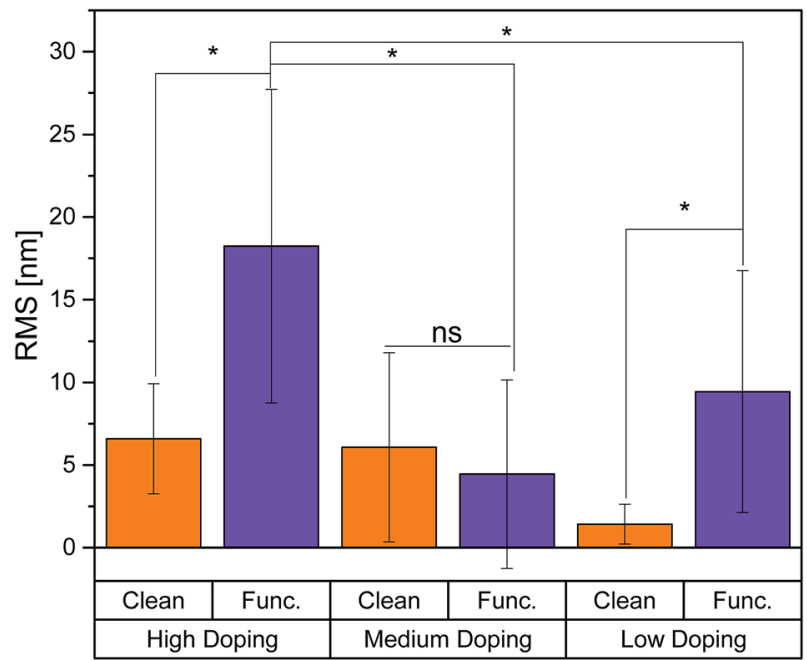

Fig. 1 The RMS was quantified using AFM. Functionalization was shown to increase roughness for the HD and LD samples, while there was no significant difference between the clean and functionalized surfaces for the MD samples.

respect to chemical functionalization. The in situ functionalization described in the Experimental section uses a short phosphonic acid adsorbate ( $n$-hexylphosphonic acid), terminates the surface with methyl groups and is intended to passivate the semiconductor surface. Functionalization in general, increased sample roughness, except for the MD samples, which showed no significant difference between clean and functionalization samples, shown in Fig. 1. For the HD samples, functionalization increased the RMS from $\sim 7 \mathrm{~nm}$ to $\sim 17 \mathrm{~nm}$. For the LD samples, functionalization increased RMS from $\sim 3 \mathrm{~nm}$ to $\sim 10 \mathrm{~nm}$. The functionalized HD samples were significantly higher than that of the MD and LD samples. No significant differences were shown between the clean samples for all different doping levels.

\section{Surface potential}

The surface potential was measured by Kelvin Probe Force Microscopy (KPFM). For all doping conditions except LD, functionalization increases the surface potential, as is shown in Fig. 2. The clean, LD surface potential increased from an initially $0.5 \mathrm{~V}$ to $0.9 \mathrm{~V}$ after UV illumination. The functionalized, LD samples resembled the PPC behavior of the clean LD samples and increased from $0.5 \mathrm{~V}$ to $0.7 \mathrm{~V}$ after UV. The MD clean samples increased from $0.5 \mathrm{~V}$ to $1.2 \mathrm{~V}$, while the MD functionalized samples showed the highest surface potential of all samples tested increasing from $2.1 \mathrm{~V}$ to $2.4 \mathrm{~V}$ after UV exposure. The HD clean samples showed no significant differences after illumination, while functionalization enhanced HD sample photoconductivity and UV light increased from $1.3 \mathrm{~V}$ to $1.7 \mathrm{~V}$.

\section{Surface chemistry}

The surface chemistry was quantitatively analyzed using XPS. For each test condition, three replicates were measured. In addition, the three spots on each replicate were analyzed to 


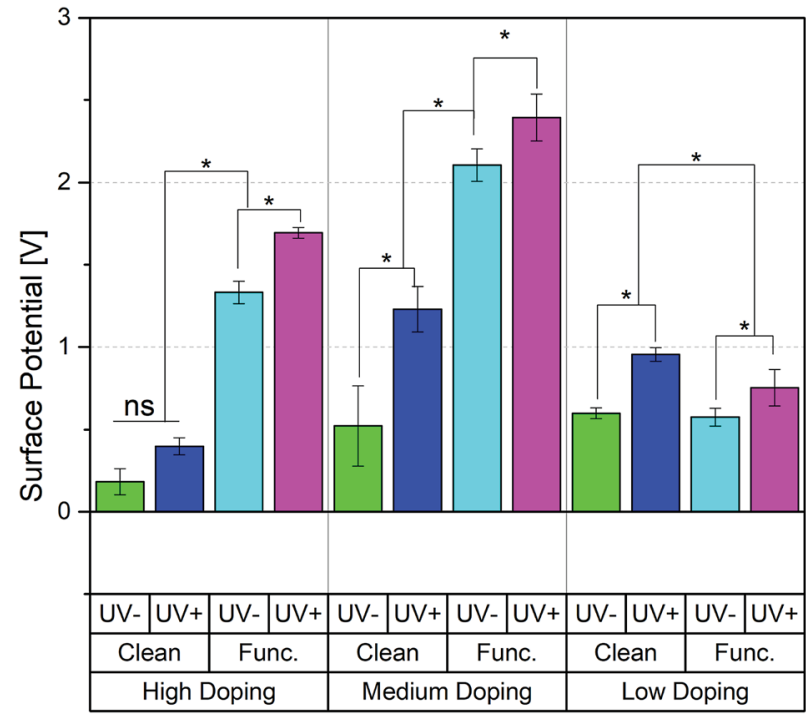

Fig. 2 The surface potential was quantified using KPFM. Functionalization was shown to increase the surface potential for all sample conditions (LD, MD, HD). Furthermore, the n-type GaN increased its surface potential upon UV exposure for all sample conditions.

obtain an average surface composition over a total of nine spots. For all materials (LD, MD, and HD), functionalization significantly increased the at\% of oxygen on the surface as shown in Table 1 . The oxygen content was shown to be significantly higher for those samples that were exposed to UV light prior to functionalization than those samples that were left in the dark. For the two highest doped materials (HD, and MD), functionalized samples that were subject to UV exposure had greater oxygen content over UV-samples. The effect was opposite for the functionalized LD samples, UV exposure was shown to decrease oxygen content, in this case. The carbon content is closely correlated to that of oxygen, though the differences between the clean and functionalized samples are decreased.

\section{Cell viability}

An Alamar Blue $(\mathrm{AB})$ assay was performed over a seven-day period. We note that the PC12 cells were never exposed to UV light and all illumination was done on samples prior to introduction into the cell culture. A significant decrease was found for all doping levels (HD, MD, LD) from day 0 (the day of cell seeding) to all the other days, shown in Fig. 3. This suggests that some cells were not completely attached to the sample or the collagen-coated Petri dish, and were removed during media aspiration. It is important to note that cell seeding was done on the semiconductor using $0.5 \mathrm{ml}$ of cell-suspension, prior to filling with cell media. After $15 \mathrm{~min}$, the culture dish was filled to its working volume with prepared cell media. In general, large differences in the $\mathrm{AB}$ reduction between samples conditions were not present, suggesting the materials show biocompatible characteristics. The MD and LD surfaces have constant $\mathrm{AB}$ levels across the seven days - while the cells are not dying they are not proliferating or showing increased metabolic activity. The same is true for the HD materials except the functionalized HD material which has slightly more $\mathrm{AB} / \mathrm{cells}$ which may reflect some increase in cellular adhesion. For the HD samples, the functionalized-UV-samples were shown to reduce significantly more $\mathrm{AB}$ than all other conditions in days 1 , 3 , and 5. Differences were also found in the MD samples on day 1 , whereby the UV exposed samples reduced less AB than their non-exposed counterparts. Additionally, there was a significant increase in $\mathrm{AB}$ reduction from all conditions in day 5 to day 7 . The LD samples showed relatively little differences from day 1 to day 7 , suggesting that cell viability was maintained throughout the experiment. The lack of increasing $\mathrm{AB}$ reduction in most conditions is explained by the addition of neuronal growth factor (NGF), which induces cellular differentiation, preventing cell growth and division, 18 hours after cell seeding.

\section{Surface chemistry and ROS production}

Intracellular reactive oxygen species (ROS) generation was quantified for the clean and functionalized samples over a 7 days period of cell culture, and summarized in Fig. 4 . It is important to note that the UV exposure to the semiconductor material was conducted minutes prior to cell seeding. Cell seeding occurred 18 hours prior to the first day of measurement (day 0). Because of this, the cellular effects are a result of the transient PPC effect previously described ${ }^{23}$ a response which is independent of cell-age due to the acute exposure at the beginning of the study. For the HD samples, there were no

Table 1 Summary of the chemical composition of the samples based on XPS analysis

\begin{tabular}{|c|c|c|c|c|c|c|c|}
\hline $\mathrm{HD}$ & UV- & Clean & $4.0 \pm 0.4$ & $6 \pm 1$ & $24.1 \pm 0.8$ & $66 \pm 1$ & 0 \\
\hline HD & UV- & Func. & $10 \pm 1$ & $7.1 \pm 0.6$ & $20.1 \pm 0.9$ & $59 \pm 2$ & $4 \pm 1$ \\
\hline HD & UV+ & Func. & $17 \pm 9$ & $10 \pm 3$ & $16 \pm 5$ & $48 \pm 12$ & $9 \pm 6$ \\
\hline MD & UV- & Clean & $4.2 \pm 0.7$ & $5.1 \pm 0.8$ & $24.9 \pm 0.5$ & $65.8 \pm 0.9$ & 0 \\
\hline MD & UV- & Func. & $9 \pm 4$ & $7 \pm 1$ & $21 \pm 3$ & $59 \pm 5$ & $4 \pm 3$ \\
\hline $\mathrm{LD}$ & UV- & Clean & $3.7 \pm 0.4$ & $5.7 \pm 0.7$ & $24.7 \pm 0.4$ & $66.9 \pm 0.7$ & 0 \\
\hline LD & UV- & Func. & $16 \pm 2$ & $10 \pm 1$ & $16 \pm 1$ & $50 \pm 2$ & $8 \pm 1$ \\
\hline LD & UV+ & Clean & $3.5 \pm 0.2$ & $4.1 \pm 0.4$ & $26.0 \pm 0.5$ & $66.4 \pm 0.8$ & 0 \\
\hline LD & UV+ & Func. & $8.0 \pm 2$ & $5 \pm 1$ & $23 \pm 1$ & $62 \pm 4$ & $2 \pm 1$ \\
\hline
\end{tabular}



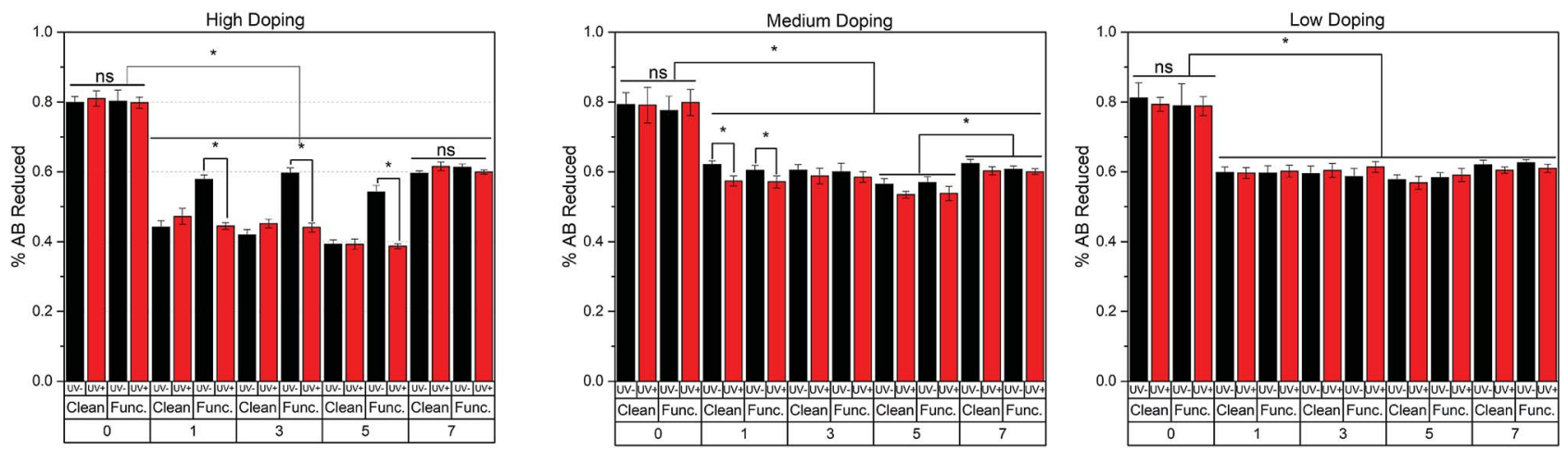

Fig. 3 Cell viability using Alamar Blue (AB) was measured across the seven-day experiment. Though day 0 saw a significant decrease in $A B$ reduction, that level was maintained throughout the experiment, verifying the biocompatible characteristics of GaN.

significant differences between samples not treated with light (UV-) and samples that were illuminated prior to cell experiments (UV+) for days 0,1 and 7. For days 3 and 5, clean HD samples that were UV exposed saw increased ROS production over non-UV exposed samples. An opposite effect was shown for functionalized HD samples, as the UV exposed samples on days 1,5 , and 7 had less ROS production than the samples which were kept in the dark. For the clean MD samples, no difference in UV exposure on days 0 and 1 was shown. For all other days, UV exposed samples decreased ROS production. For the functionalized MD samples, no difference in UV exposure on days 0 and 3 was shown. On day 1 , UV exposed samples were higher than those not exposed. The effect was opposite on days 5 and 7. For clean Low Doping (LD) samples, no difference was found between UV exposure on days 0 and 3 . The UV exposed samples produced more ROS on days 5 and 7 . The LD functionalized samples on days 0,1 , and 7 saw no difference between $\mathrm{UV}+$ and UV- conditions. On days 3 and 5, UV exposed samples produced more ROS than those left in the dark. Overall, this relationship between surface chemistry and the amount of ROS produced, is difficult to interpret if conducted independently. Thus, we attempted make this easier by categorizing the ROS production with the surface chemistry (gallium, nitrogen, oxygen, carbon, and phosphorous content).

As previously stated, the three sample types with the highest oxygen, carbon, and phosphorous content (indicative of chemical functionalization) are HD-functionalized-UV+, MD-functionalized-UV+, and LD-functionalized-UV-. These 3 materials are denoted with a triangle in Fig. 4. This figure shows samples with the higher at $\%$ of $\mathrm{C}, \mathrm{O}$, and $\mathrm{P}$ (characteristics of functionalization), generally show (7 out of 8 instances) a lesser amount of ROS species produced. The one exception to this trend is the MD-functionalized-UV+ samples on day 1 , which showed a higher ROS production despite it having a higher average at $\%$ of $\mathrm{O}$ and $\mathrm{C}$ than its UV- counterpart (denoted by the upside down triangle).

Taken as a whole, we can begin to make some conclusions as to how the surface chemistry relates to the production of ROS species when PC12 cells are cultured in the presence of the semiconductor surfaces. In this regard, the following with respect to the n-type Ga-polar samples tested can be summarized:

(1) For the two highest doped samples (HD and MD), UV exposure prior to chemical functionalization increased the
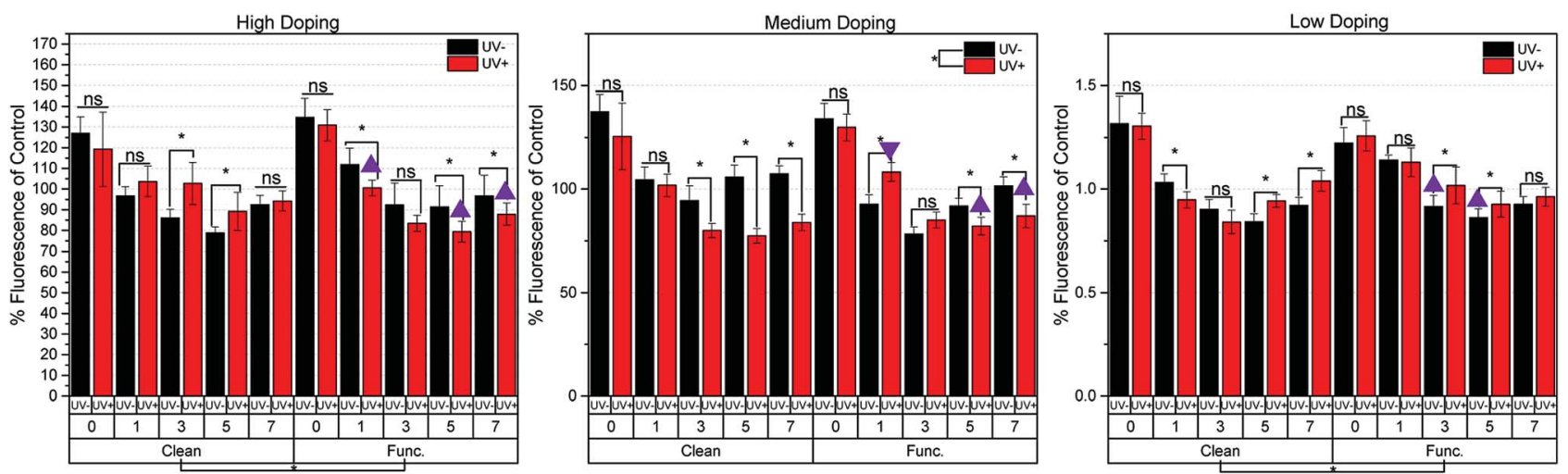

Fig. 4 ROS production was measured throughout the seven-day experiment. These results are supported by the surface chemistry results. Three conditions which have the highest oxygen, carbon, and phosphorous content (indicative of chemical functionalization) are HD-functionalized-UV+, MD-functionalized-UV+, and LD-functionalized-UV-. These conditions are noted with a triangle. Triangle $=$ lesser amount of ROS produced than their UV or non-UV counterpart. Upside-down triangle = greater amount of ROS produced than the UV-counterpart. This suggests that chemical functionalization inhibits ROS production. 
oxygen and carbon surface content as compared to samples left in the dark. The opposite trend was found for the LD samples. This supports the notion that chemical passivation may be enhanced by charging the substrate via UV exposure prior to etching. We note that there might be minimum critical doping limit for which UV exposure shows no enhancement.

(2) Surface analysis verified chemical attachment of the functionalized samples. Samples with a greater amount of adsorbates (C, O, and $\mathrm{P}$ ) on the surface correspond with significantly less at $\% \mathrm{~N}$ and Ga on the surface. It was shown that these samples that contain higher $\mathrm{C}, \mathrm{O}$, and $\mathrm{P}$ species generate less ROS when exposed to PC12 cells.

(3) In this work we intentionally did not use UV light as a way of generating ROS in solution in the presence of water and semiconductor material. However, UV exposure which changes the surface charge plays a direct role in the chemical species formed on the surface during chemical functionalization. These species were shown to have a direct passivating effect on the generation of ROS by PC12 cells in culture.

\section{Changes in $\left[\mathrm{Ca}^{2+}\right]_{\mathrm{i}}$ \& ROS production}

In general, some relationship exists between a material doping level (which largely affects surface charge buildup) and chemical functionality with respect to the amount of ROS produced and the resulting changes in $\left[\mathrm{Ca}^{2+}\right]_{\mathrm{i}}$. The highly doped samples showed moderate changes in surface potential before and after UV exposure, Fig. 2. The significant changes in ROS production were then cross-referenced with significant $\left[\mathrm{Ca}^{2+}\right]_{\mathrm{i}}$ changes, and symbols were drawn to show direct proportionality, inverse proportionality, or no proportionality, Fig. 5. The clean HD samples on days 3 and 5 showed an inverse relationship between ROS production and $\left[\mathrm{Ca}^{2+}\right]_{\mathrm{i}}$ (represented by a filled green star). The chemistry of the surface was shown to affect how ROS production translates to $\left[\mathrm{Ca}^{2+}\right]_{\mathrm{i}}$ changes, in that a passivating effect was shown on days 1 and 5 of functionalized HD samples (changes in ROS production did not result in significant $\left[\mathrm{Ca}^{2+}\right]_{\mathrm{i}}$ changes). Day 7 of the HD functionalized samples showed a direct relationship (more ROS production $=$ higher intracellular $\left[\mathrm{Ca}^{2+}\right]_{\mathrm{i}}$ ).

A similar effect was even more pronounced when looking at the MD samples, which showed the greatest changes in surface potential of all samples tested. For the clean MD samples, direct proportionality was displayed on days 3 and 7 , while day 5 showed no significant changes in $\left[\mathrm{Ca}^{2+}\right]_{\mathrm{i}}$ as a result of changes in ROS production. The functionalized MD samples had the highest surface charge (and largest surface potential changes between $\mathrm{UV}+$ and $\mathrm{UV}-$ ), and thus showed inverse proportionality on days 1,5 , and 7 . The LD samples showed the least amount of surface charge buildup after UV exposure (Fig. 2), and thus did not show a strong relationship between doping and chemistry versus ROS production and $\left[\mathrm{Ca}^{2+}\right]_{\mathrm{i}}$ changes, Fig. 5. The only significant relation is for the clean LD samples on day 7 , which showed direct proportionality, an outlier of the other data, though notably with large error involved. The clean LD samples on days 1 and 5, as well as the functionalized LD samples on days 3 and 5 showed no significant relationship between the amount of ROS produced and resulting changes in intracellular $\left[\mathrm{Ca}^{2+}\right]_{\mathrm{i}}$.

The above observations lead us to the question of what are the possible mechanisms that provide for: (1) ROS production running proportional to $\left[\mathrm{Ca}^{2+}\right]_{i}$; (2) ROS production running inversely proportional to $\left[\mathrm{Ca}^{2+}\right]_{\mathrm{i}}$; and (3) ROS production being independent of $\left[\mathrm{Ca}^{2+}\right]_{\mathrm{i}}$ ? In previous studies conducted by our group, we showed that when n-type Ga-polar GaN is exposed to UV light, and subsequently placed in contact with PC12 cells, cell populations show an increased $\left[\mathrm{Ca}^{2+}\right]_{\mathrm{i}}$. Since the measurement is done immediately after the charging of the surface this assay can be used to assess cell stimulation. Furthermore, in the same work we showed that intentionally introducing $\mathrm{Ga}^{3+}$ ions in solution can decreases the $\left[\mathrm{Ca}^{2+}\right]_{\mathrm{i}}{ }^{25}$ In the present long term study, where the Ca assay is used to quantify mechanical cell responses over a period of days, rather than a stimulation response, we can make the following conclusions:

(1) ROS production is proportional to $\left[\mathrm{Ca}^{2+}\right]_{\mathrm{i}}$ : the functionalized samples with high $\mathrm{C}, \mathrm{O}$, and $\mathrm{P}$ at\%, generate less
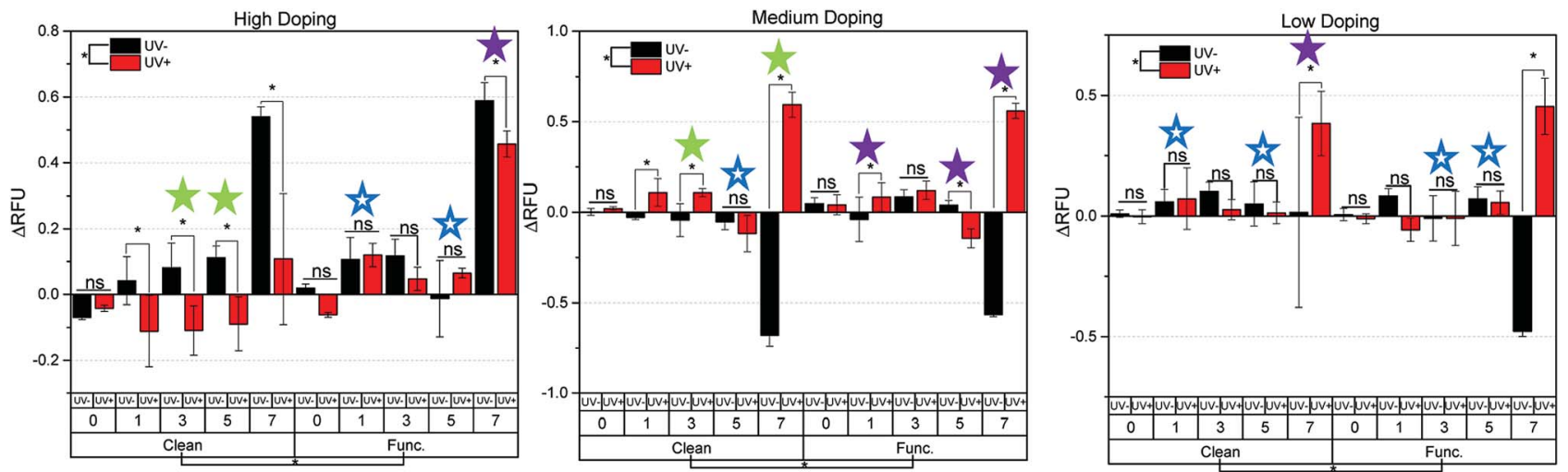

Fig. 5 The changes in $\left[\mathrm{Ca}^{2+}\right]_{i}$ with respect to material doping (HD, MD, and LD), chemistry (clean, and functionalized), and UV exposure were examined. These results correlated closely to the production of ROS in culture. The symbols are drawn to denote the following: filled green star $=\mathrm{ROS}$ production is inverse to $\left[\mathrm{Ca}^{2+}\right]_{\mathrm{i}}$, filled purple star $=\mathrm{ROS}$ production is proportional to $\left[\mathrm{Ca}^{2+}\right]_{\mathrm{i}}$, empty blue star $=$ significant changes in ROS production did not significantly affect intracellular Ca concentration (no significant relation). 
ROS species when placed in cell culture than their clean counterparts. This is intuitive, as the hydrocarbon chains in the hexylphosphonic acid are hydrophobic in nature, and presumably decrease water contact with the material. For the same reason, this functionalization will likely decrease leaching effects, which has the effect of decreasing intracellular $\left[\mathrm{Ca}^{2+}\right]_{\mathrm{i}}$. Furthermore, an additive effect also in play is the cytotoxic effects of ROS, which has a contributing role in directly increasing $\left[\mathrm{Ca}^{2+}\right]_{\mathrm{i}}$ which has been shown previously by others. ${ }^{32}$

(2) ROS production is inversely proportional to $\left[\mathrm{Ca}^{2+}\right]_{\mathrm{i}}$ : the clean samples show significantly higher at\% Ga and $\mathrm{N}$ (and lower at\% $\mathrm{C}$ and $\mathrm{O}$ ) on the surface than those functionalized. Because of these exposed surfaces, the formation of galliumbased leachates in solution is more likely to occur. As previously described: higher amounts of gallium leachates = lower amounts of $\left[\mathrm{Ca}^{2+}\right]_{\mathrm{i}} \cdot{ }^{25}$ Thus in this mechanism, the effect of gallium leaching over the seven-day experiment is shown to overpower the mechanisms described in case 1 .

(3) ROS production is independent of $\left[\mathrm{Ca}^{2+}\right]_{\mathrm{i}}$ : in this case, there are competing effects of the mechanisms described in case 1 and case 2 . One can observe that this occurred most often in the case of the low doping material. This likely occurred due to low charge carrier concentration relative to the other samples, resulting in small changes in surface potential before and after UV exposure. This mechanism was muddled by the competing effect of leaching over the seven-day experiment. Previous studies have shown GaN to be stable in even harsh conditions, and no significant leaching over a 2 days period. ${ }^{25,33}$

This study, up to this point, has largely ignored the cellular mechanics and processes which might be involved in cellular stimulation while instead focusing on the material's properties (PPC, doping, chemistry, etc.). It is important to note however that both, intracellular ROS production and calcium release are important intracellular signaling molecules, which are present in various organelles in the cell, and often have either self-amplifying or deteriorative relationships depending on not only the organelle function, but also the metabolic state of the cell. ${ }^{34}$ Furthermore, it is known that the levels of intracellular ROS are regulated by and modulate the intracellular calcium levels, thus generating more complexity in the coordination and control of intracellular signaling. Görlach and colleagues have recently published a review discussing the relationship of calcium and ROS production. ${ }^{34}$ This review along with the included literature and our results, suggest that there may be a complex interplay between these signaling systems among different organelles. ${ }^{35-38}$ Identifying those organelles and cellular pathways will be required for the future fine tuning of specific cellular signaling networks.

\section{Conclusions}

In summary, we examined the effect of GaN doping and chemistry on the behavior of PC12 cells. In the process, we were able to provide the answers to the following important questions:
(1) How can PPC change the surface chemistry prior to chemical functionalization of samples with different doping levels?

PPC, through an increase in surface potential, enhanced the chemical functionalization of hexylphosphonic (higher at\% of $\mathrm{C}, \mathrm{O}$, and $\mathrm{P}$ ) on the surface over samples which did not experience $\mathrm{PPC}$.

(2) How does the concentration of dopants affect PPCinduced surface changes and subsequent alteration of $\left[\mathrm{Ca}^{2+}\right]_{\mathrm{i}}$ ?

Samples that had high concentrations of charge carriers (HD and MD), in general, showed a better ability to cause changes in $\left[\mathrm{Ca}^{2+}\right]_{\mathrm{i} \text {. }}$.

(3) How does chemical functionalization affect PPC?

In this study, chemical functionalization combined with PPC was shown to passivate the surface in such a way to as to screen the amount of $\mathrm{Ga}$ and $\mathrm{N}$ atoms near the surface. This minimizes ROS production attributable to leachates, while increasing direct cellular charge transfer from material to cell, both of which have the same additive effect of increasing cellular stimulation.

The presented work also revealed that there are likely competing mechanisms of PPC-induced $\left[\mathrm{Ca}^{2+}\right]_{\mathrm{i}}$ changes. The possible mechanisms we outlined are related to the stability of the material and our ability to control it via surface charge aided functionalization. We plan to explore how to control these competing mechanisms in future studies using adsorbates with different chemical functionality.

\section{Conflicts of interest}

There are no conflicts to declare.

\section{Acknowledgements}

We thank Army Research Office under W911NF-15-1-0375 for support of this work. Partial financial support from NSF (DMR1312582 and ECCS-1653383) is greatly appreciated. This work was performed in part at the Chapel Hill Analytical and Nanofabrication Laboratory, CHANL, a member of the North Carolina Research Triangle Nanotechnology Network, RTNN, which is supported by the National Science Foundation, Grant ECCS1542015, as part of the National Nanotechnology Coordinated Infrastructure, NNCI.

\section{References}

1 R. Kirste, N. Rohrbaugh, I. Bryan, Z. Zryan, R. Collazo and A. Ivanisevic, Electronic Biosensors Based on III-Nitride Semiconductors, Annu. Rev. Anal. Chem., 2015, 8, 149-169.

2 S. A. Chambers, Ferromagnetism in doped thin-film oxide and nitride semiconductors and dielectrics, Surf. Sci. Rep., 2006, 61, 345-381.

3 M. H. Park, Y. H. Lee, H. J. Kim, Y. J. Kim, T. Moon, K. D. Kim, J. Müller, A. Kersch, U. Schroeder and T. Mikolajick, Ferroelectricity and Antiferroelectricity of Doped Thin $\mathrm{HfO}_{2}$-Based Films, Adv. Mater., 2015, 27, 18111831. 
4 K. Hantanasirisakul, M. Q. Zhao, P. Urbankowski, J. Halim, B. Anasori, S. Kota, C. E. Ren, M. W. Barsoum and Y. Gogotsi, Fabrication of $\mathrm{Ti}_{3} \mathrm{C}_{2} \mathrm{~T}_{\mathrm{x}}$ MXene transparent thin films with tunable optoelectronic properties, Adv. Electron. Mater., 2016, 2, 1600050.

5 B. Russ, M. J. Robb, B. C. Popere, E. E. Perry, C.-K. Mai, S. L. Fronk, S. N. Patel, T. E. Mates, G. C. Bazan and J. J. Urban, Tethered tertiary amines as solid-state n-type dopants for solution-processable organic semiconductors, Chem. Sci., 2016, 7, 1914-1919.

6 Y. S. Rim, S.-H. Bae, H. Chen, J. L. Yang, J. Kim, A. M. Andrews, P. S. Weiss, Y. Yang and H.-R. Tseng, Printable ultrathin metal oxide semiconductor-based conformal biosensors, ACS Nano, 2015, 9, 12174-12181.

7 E. Manikandan, V. Murugan, G. Kavitha, P. Babu and M. Maaza, Nanoflower rod wire-like structures of dual metal ( $\mathrm{Al}$ and $\mathrm{Cr}$ ) doped $\mathrm{ZnO}$ thin films: Structural, optical and electronic properties, Mater. Lett., 2014, 131, 225-228.

8 Y. Morikawa, S. Nagano, K. Watanabe, K. Kamata, T. Iyoda and T. Seki, Optical alignment and patterning of nanoscale microdomains in a block copolymer thin film, Adv. Mater., 2006, 18, 883-886.

9 Y. Xia, J. A. Rogers, K. E. Paul and G. M. Whitesides, Unconventional methods for fabricating and patterning nanostructures, Chem. Rev., 1999, 99, 1823-1848.

10 M. Hetzl, J. Wierzbowski, T. Hoffmann, M. Kraut, V. Zuerbig, C. E. Nebel, K. Müller, J. J. Finley and M. Stutzmann, GaN Nanowire Arrays for Efficient Optical Read-Out and Optoelectronic Control of NV Centers in Diamond, Nano Lett., 2018, 3651-3660.

11 C. S. Lao, Y. Li, C. P. Wong and Z. L. Wang, Enhancing the electrical and optoelectronic performance of nanobelt devices by molecular surface functionalization, Nano Lett., 2007, 7, 1323-1328.

12 I. Salzmann, G. Heimel, M. Oehzelt, S. Winkler and N. Koch, Molecular electrical doping of organic semiconductors: fundamental mechanisms and emerging dopant design rules, Acc. Chem. Res., 2016, 49, 370-378.

13 N. Martino, P. Feyen, M. Porro, C. Bossio, E. Zucchetti, D. Ghezzi, F. Benfenati, G. Lanzani and M. R. Antognazza, Photothermal cellular stimulation in functional biopolymer interfaces, Sci. Rep., 2015, 5, 8911.

14 M. Mamat, M. Sahdan, Z. Khusaimi, A. Z. Ahmed, S. Abdullah and $M$. Rusop, Influence of doping concentrations on the aluminum doped zinc oxide thin films properties for ultraviolet photoconductive sensor applications, Opt. Mater., 2010, 32, 696-699.

15 M. Cole, P. Joshi, M. Ervin, M. Wood and R. Pfeffer, The influence of $\mathrm{Mg}$ doping on the materials properties of $\mathrm{Ba}_{1-\mathrm{x}} \mathrm{Sr}_{\mathrm{x}} \mathrm{TiO} 3$ thin films for tunable device applications, Thin Solid Films, 2000, 374, 34-41.

16 C. R. Ryder, J. D. Wood, S. A. Wells, Y. Yang, D. Jariwala, T. J. Marks, G. C. Schatz and M. C. Hersam, Covalent functionalization and passivation of exfoliated black phosphorus via aryl diazonium chemistry, Nat. Chem., 2016, 8, 597.
17 P. Solís-Fernández, M. A. Bissett, M. Tsuji and H. Ago, Tunable doping of graphene nanoribbon arrays by chemical functionalization, Nanoscale, 2015, 7, 3572-3580.

18 T.-H. Yoo, H. G. Moon, B.-Y. Wang, B.-I. Sang, B. Angadi, Y.-J. Oh, W. K. Choi, C.-Y. Kang and D. K. Hwang, InGaZnO transistor based on porous $\mathrm{Ag}$ nanowirefunctionalized gate electrode for detection of bio-relevant molecules, Sens. Actuators, B, 2018, 254, 36-43.

19 Y. Chen, Y. Xianyu, J. Wu, B. Yin and X. Jiang, Click chemistry-mediated nanosensors for biochemical assays, Theranostics, 2016, 6, 969.

20 M. A. Colicos, B. E. Collins, M. J. Sailor and Y. Goda, Remodeling of synaptic actin induced by photoconductive stimulation, Cell, 2001, 107, 605-616.

21 J. Campbell, D. Singh, G. Hollett, S. M. Dravid, M. J. Sailor and J. Arikkath, Spatially selective photoconductive stimulation of live neurons, Front. Cell. Neurosci., 2014, 8, 142.

22 Y. Goda and M. A. Colicos, Photoconductive stimulation of neurons cultured on silicon wafers, Nat. Protoc., 2006, 1, 461.

23 P. J. Snyder, R. Kirste, R. Collazo and A. Ivanisevic, Persistent Photoconductivity, Nanoscale Topography, and Chemical Functionalization Can Collectively Influence the Behavior of PC12 Cells on Wide Bandgap Semiconductor Surfaces, Small, 2017, 1700481.

24 X. Dang, C. Wang, E. Yu, K. Boutros and J. Redwing, Persistent photoconductivity and defect levels in n-type AlGaN/GaN heterostructures, Appl. Phys. Lett., 1998, 72, 2745-2747.

25 P. J. Snyder, P. Reddy, R. Kirste, D. R. LaJeunesse, R. Collazo and A. Ivanisevic, Noninvasive Stimulation of Neurotypic Cells Using Persistent Photoconductivity of Gallium Nitride, ACS Omega, 2018, 3, 615-621.

26 M. J. Berridge, M. D. Bootman and H. L. Roderick, Calcium: Calcium signalling: dynamics, homeostasis and remodelling, Nat. Rev. Mol. Cell Biol., 2003, 4, 517.

27 R. D. Adams, B. Gupta and A. B. Harkins, Validation of electrical stimulation models: intracellular calcium measurement in three-dimensional scaffolds, $J$. Neurophysiol., 2017, 118, 1415-1424.

$28 \mathrm{H}$. Hong and G.-Q. Liu, Protection against hydrogen peroxide-induced cytotoxicity in PC12 cells by scutellarin, Life Sci., 2004, 74, 2959-2973.

29 R. Collazo, S. Mita, A. Aleksov, R. Schlesser and Z. Sitar, Growth of Ga-and N-polar gallium nitride layers by metalorganic vapor phase epitaxy on sapphire wafers, $J$. Cryst. Growth, 2006, 287, 586-590.

30 F. Kaess, S. Mita, J. Xie, P. Reddy, A. Klump, L. H. HernandezBalderrama, S. Washiyama, A. Franke, R. Kirste and A. Hoffmann, Correlation between mobility collapse and carbon impurities in Si-doped GaN grown by low pressure metalorganic chemical vapor deposition, J. Appl. Phys., 2016, 120, 105701.

31 P. Reddy, I. Bryan, Z. Bryan, W. Guo, L. Hussey, R. Collazo and Z. Sitar, The effect of polarity and surface states on the Fermi level at III-nitride surfaces, J. Appl. Phys., 2014, 116, 123701. 
32 A. Haase, S. Rott, A. Mantion, P. Graf, J. Plendl, A. F. Thünemann, W. P. Meier, A. Taubert, A. Luch and G. Reiser, Effects of silver nanoparticles on primary mixed neural cell cultures: uptake, oxidative stress and acute calcium responses, Toxicol. Sci., 2012, 126, 457-468.

33 C. A. Chapin, R. A. Miller, K. M. Dowling, R. Chen and D. G. Senesky, InAlN/GaN high electron mobility micropressure sensors for high-temperature environments, Sens. Actuators, A, 2017, 263, 216-223.

34 A. Görlach, K. Bertram, S. Hudecova and O. Krizanova, Calcium and ROS: a mutual interplay, Redox Biol., 2015, 6, 260-271.
35 R. Sano and J. C. Reed, ER stress-induced cell death mechanisms, Biochim. Biophys. Acta, Mol. Cell Res., 2013, 1833, 3460-3470.

36 A. Görlach, P. Klappa and D. T. Kietzmann, The endoplasmic reticulum: folding, calcium homeostasis, signaling, and redox control, Antioxid. Redox Signaling, 2006, 8, 1391-1418.

37 V. Adam-Vizi and A. A. Starkov, Calcium and mitochondrial reactive oxygen species generation: how to read the facts, $J$. Alzheimer's Dis., 2010, 20, S413-S426.

38 X. Li, P. Fang, J. Mai, E. T. Choi, H. Wang and X.-f. Yang, Targeting mitochondrial reactive oxygen species as novel therapy for inflammatory diseases and cancers, J. Hematol. Oncol., 2013, 6, 19. 